\title{
Non degeneracy of critical points of the Robin function with respect to deformations of the domain
}

\author{
Anna Maria Micheletti* Angela Pistoia ${ }^{\dagger}$
}

June 22, 2018

\begin{abstract}
We show a result of genericity for non degenerate critical points of the Robin function with respect to deformations of the domain.
\end{abstract}

Keywords: Robin function, non degenerate critical points, generic property

AMS subject classification: 35J08, 35J25, 35G30

\section{Introduction}

Let $\Omega$ be a smooth bounded domain in $\mathbb{R}^{N}, N \geq 2$. The Green function of the Laplace operator vanishing at the boundary $\partial \Omega$ is of the form

$$
G_{y}(x)=\frac{1}{\omega_{N}}\left[\Gamma_{y}(x)-H_{y}(x)\right], x, y \in \Omega,
$$

with where $\omega_{N}$ denotes the surface area of the unit sphere in $\mathbb{R}^{N}$. The singular part $\Gamma_{y}$ is given by $\Gamma_{y}(x)=$ $\Gamma(|x-y|)$

$$
\Gamma(|x-y|)=-\ln |x-y| \text { if } N=2 \quad \text { and } \quad \Gamma(|x-y|)=\frac{1}{N-2}|x-y|^{2-N} \text { if } N \geq 3 .
$$

The regular part $H_{y}$ is a harmonic function with the same boundary value as the singular part, i.e. for any $y \in \Omega$

$$
\left\{\begin{array}{l}
\Delta_{x} H_{y}(x)=0 \quad \text { if } x \in \Omega \\
H_{y}(x)=\Gamma_{y}(x) \quad \text { if } x \in \partial \Omega .
\end{array}\right.
$$

The Robin function of $\Omega$ is defined by $t(x):=t^{\Omega}(x):=H_{x}(x), x \in \Omega$.

This function plays an important role in various fields of the mathematics, e.g., geometric function theory, capacity theory, concentration problems (see [2] and the references therein).

In particular, existence and uniqueness of solutions of some critical problems is strictly dependent on the non degeneracy of critical points of the Robin function (see, for example, 1, 7, 12, 14. Non degenerate critical points of the Robin function plays also a crucial role in studying existence and uniqueness of solutions of the Gelfand's problem (see for example [3, 5, 6, 9, 11).

As far as we know, the only results about non degeneracy of critical points of the Robin function are in [4] and 10 . In 4 the authors show that the Robin function of a smooth bounded and convex domain of $\mathbb{R}^{2}$ has a unique critical point which is non degenerate. In [10] the author proves that the origin is a non degenerate critical point of the Robin function of a smooth bounded domain of $\mathbb{R}^{N}$ which is symmetric with respect to the origin and convex in any directions $x_{1}, \ldots, x_{N}$.

Here we prove that for most domains the critical points of the Robin function are non degenerate.

\footnotetext{
*Dipartimento di Matematica Applicata "U.Dini", Università di Pisa, Via Filippo Buonarroti, 156127 Pisa, Italy E-mail address: a.micheletti@dma.unipi.it

${ }^{\dagger}$ Dipartimento di Scienze di Base e Applicate per l’Ingegneria, Università di Roma "La Sapienza", via A. Scarpa 16, 00161 Roma, Italy. E-mail address: pistoia@dmmm.uniroma1.it
} 
Let $\Omega \subset \mathbb{R}^{N}$ be a domain of class $C^{k}$ with $k \geq 4$ and $N \geq 2$. We consider the domain $\Omega_{\theta}:=(I+\theta) \Omega$ given by the deformation $I+\theta$. Here $I$ is the identity map on $\mathbb{R}^{N}$.

We are interested in studying the non degeneracy of the critical points of the Robin function of the domain $\Omega_{\theta}$ with respect to the parameter $\theta$.

Let $\mathfrak{E}^{k}$ be the vector space of all the $C^{k}$ applications $\theta: \mathbb{R}^{N} \rightarrow \mathbb{R}^{N}$ such that

$$
\|\theta\|_{k}:=\sup _{x \in \mathbb{R}^{N}} \max _{0 \leq|\alpha| \leq k}\left|\frac{\partial^{\alpha} \theta_{i}(x)}{\partial x_{1}^{\alpha_{1}} \ldots \partial x_{N} \alpha_{N}}\right|<+\infty .
$$

$\mathfrak{E}^{k}$ is a Banach space equipped with the norm $\|\cdot\|_{k}$. Let $\mathfrak{B}_{\rho}:=\left\{\theta \in \mathfrak{E}^{k}:\|\theta\|_{k} \leq \rho\right\}$ be the ball in $\mathfrak{E}^{k}$ centered at 0 with radius $\rho$. We will prove the following result.

Theorem 1.1. The set $\mathfrak{A}:=\left\{\theta \in \mathfrak{B}_{\rho}\right.$ : all the critical points of the Robin function of the domain $\Omega_{\theta}$ are non degenerate $\}$ is a residual (hence dense) subset of $\mathfrak{B}_{\rho}$, provided $\rho$ is small enough.

To get Theorem 1.1 we use an abstract transversality theorem previously used by Quinn [13, Saut and Temam [15] and Uhlenbeck [16]. The strategy in our work is similar to the one used by Saut and Temam in [15] to get some generic property with respect to the domain of the solutions to certain semilinear elliptic equations. In our case we need some new delicate estimates which involve the derivative of Robin function with respect to the variation of the domain.

The paper is organized as follows. In Section 2 we set the problem and we prove the main result. All the technical results are proved in Section 3 and in Section 4.

\section{Setting of the problem and proof of the main result}

First of all let us recall some useful properties of the Robin function (see [2]).

Remark 2.1. If $\Omega$ is of class $C^{2, \alpha}$ then the Robin function $t \in C^{2, \alpha}(\bar{\Omega})$ and it holds

$$
\nabla t(x)=2 \nabla_{x} H_{y}(x)_{\left.\right|_{y=x}} \quad \text { and } \quad \frac{\partial^{2} t}{\partial x_{i} \partial x_{j}}(x)=4 \frac{\partial^{2} H_{y}}{\partial x_{i} \partial x_{j}}(x)_{\left.\right|_{y=x}}
$$

Given $\Omega \subset \mathbb{R}^{N}, N \geq 2$ of class $C^{k}$ with $k \geq 3$, we consider the domain $\Omega_{\theta}:=(I+\theta) \Omega$ with $\theta \in \mathfrak{B}_{\rho}$. It is well known that we can choose $\rho$ positive and small enough such that if $\theta \in \mathfrak{B}_{\rho}$ then the map $I+\theta: \bar{\Omega} \rightarrow(I+\theta) \bar{\Omega}$ is a diffeomorphism of class $C^{k}$. We set $I+\gamma=(I+\theta)^{-1}$.

Remark 2.2. Since, by definition $(I+\theta) \circ(I+\gamma)=I$ we have that $\gamma(z)=-\theta(z+\gamma(z))$. Moreover, it holds

$$
\left[I+\theta^{\prime}(z+\gamma(z))\right]\left(h+\gamma^{\prime}(z)(h)\right)=h \quad \forall h \in \mathbb{R}^{N} .
$$

Then we have

$$
\left[I+\theta^{\prime}(z+\gamma(z))\right] \circ \gamma^{\prime}(z)=-\theta^{\prime}(z+\gamma(z))
$$

which implies

$$
\gamma^{\prime}(z)=-\left[I+\theta^{\prime}(x)\right]^{-1} \circ \theta^{\prime}(x)=\sum_{i \geq 0}(-1)^{i+1}\left[\theta^{\prime}(x)\right]^{i+1} \quad \text { where } x=z+\gamma(z) .
$$

Moreover by (6)

$$
\left[I+\theta^{\prime}(z+\gamma(z))\right] \circ \gamma^{\prime \prime}(z)(h)(k)=-\theta^{\prime \prime}(z+\gamma(z))\left(h+\gamma^{\prime}(z)(h)\right)\left(k+\gamma^{\prime}(z)(k)\right) \quad \forall h, k \in \mathbb{R}^{N}
$$

Then we have if $x=z+\gamma(z)$

$$
\begin{aligned}
& \gamma^{\prime \prime}(z)(h)(k)=\sum_{i \geq 0}(-1)^{i}\left[\theta^{\prime}(x)\right]^{i}\left[-\theta^{\prime \prime}(x)\left(h+\gamma^{\prime}(z)(h)\right)\left(k+\gamma^{\prime}(z)(k)\right)\right] \\
& =-\theta^{\prime \prime}(x)\left(h+\gamma^{\prime}(z)(h)\right)\left(k+\gamma^{\prime}(z)(k)\right)-\sum_{i \geq 1}(-1)^{i}\left[\theta^{\prime}(x)\right]^{i}\left[\theta^{\prime \prime}(x)\left(h+\gamma^{\prime}(z)(h)\right)\left(k+\gamma^{\prime}(z)(k)\right)\right] .
\end{aligned}
$$


Given $y \in \Omega_{\theta}$, we consider the unique function $v_{y}^{\theta}$ solution of the problem

$$
\left\{\begin{array}{lr}
\Delta_{z} v_{y}^{\theta}(z)=0 & \text { if } z \in \Omega_{\theta} \\
v_{y}^{\theta}(z)=\Gamma(|z-y|) & \text { if } z \in \partial \Omega_{\theta} .
\end{array}\right.
$$

More precisely, $v_{y}^{\theta}$ is the regular part of the Green's function of the domain $\Omega_{\theta}$. We have that $v_{y}^{\theta} \in C^{2, \alpha}\left(\bar{\Omega}_{\theta}\right)$ because $k \geq 3$. If $\xi \in \Omega$ is such that $\xi+\theta(\xi)=y$, we define the function $\widetilde{v}_{\xi}^{\theta} \in C^{2, \alpha}(\bar{\Omega})$ by

$$
\widetilde{v}_{\xi}^{\theta}(x):=v_{y}^{\theta}(x+\theta(x))=v_{y}^{\theta}(z) .
$$

The function $\widetilde{v}_{\xi}^{\theta}$ is the unique solution of the following problem

$$
\left\{\begin{array}{l}
\left.\sum_{i, j, s=1}^{N} \frac{\partial^{2} \widetilde{v}_{\xi}^{\theta}}{\partial x_{i} \partial x_{j}}\right|_{x}\left[\delta_{i s}+\left.\frac{\partial \gamma_{i}}{\partial z_{s}}\right|_{x+\theta(x)}\right]\left[\delta_{j s}+\left.\frac{\partial \gamma_{j}}{\partial z_{s}}\right|_{x+\theta(x)}\right]+\left.\left.\sum_{j, s=1}^{N} \frac{\partial \widetilde{v}_{\xi}^{\theta}}{\partial x_{j}}\right|_{x} \frac{\partial^{2} \gamma_{j}}{\partial z_{s}^{2}}\right|_{x+\theta(x)}=0 \quad \text { if } x \in \Omega \\
\widetilde{v}_{\xi}^{\theta}(x)=\Gamma(|x-\xi+\theta(x)-\theta(\xi)|)
\end{array}\right.
$$

When $\theta=0$ we obviously have that $\widetilde{v}_{\xi}^{0}$ is the unique solution of the problem

$$
\left\{\begin{array}{lr}
\Delta_{x} \widetilde{v}^{0}=0 & \text { if } x \in \Omega \\
\widetilde{v}_{\xi}^{0}(x)=\Gamma(|x-\xi|) & \text { if } x \in \partial \Omega .
\end{array}\right.
$$

Remark 2.3. It is easy to see that there exists a $C^{3}$-extension of the function $\Gamma(|z-y|)$ for $z \in \partial \Omega_{\theta}$ on the domain $\Omega_{\theta}, z \rightarrow \Gamma(|z-y|) \chi_{d}(|z-y|)$. Here the smooth cut off function $\chi_{d}$ is such that

$$
\chi_{d}(s)=0 \text { if } 0<s<d, \chi_{d}(s)=1 \text { if } s>2 d,\left|\chi^{\prime}(s)\right|<\frac{c}{d},\left|\chi^{\prime \prime}(s)\right|<\frac{c}{d^{2}}
$$

where $d=\operatorname{dist}\left(y, \partial \Omega_{\theta}\right) / 3$, for some constant $c>1$. get

Since $\widetilde{v}_{\xi}^{\theta}$ solves (10), by maximum principle and standard elliptic regularity theory (see Theorem 6.6, [8]) we

$$
\left\|\widetilde{v}_{\xi}^{\theta}\right\|_{C^{2, \alpha}(\bar{\Omega})} \leq c\left[\left\|\widetilde{v}_{\xi}^{\theta}\right\|_{C^{0}(\bar{\Omega})}+\|\varphi\|_{C^{2, \alpha}(\bar{\Omega})}\right] \leq c\left[\sup _{x \in \partial \Omega} \Gamma(|x-\xi+\theta(x)-\theta(\xi)|)+\|\varphi\|_{C^{2, \alpha}(\bar{\Omega})}\right],
$$

where

$$
\varphi(x):=\Gamma(|x-\xi+\theta(x)-\theta(\xi)|) \chi_{d}(|x-\xi+\theta(x)-\theta(\xi)|) .
$$

It is important to point out that by standard regularity theory (see Theorem 6.6, [8]) we also get that $\widetilde{v}_{\xi}^{\theta} \in$ $C^{3, \alpha}(\bar{\Omega})$ if $k \geq 4$.

Let us establish some properties of the function $\widetilde{v}_{\xi}^{\theta}$.

It is useful to point out that when $\theta=0$, for any $p=1, \ldots, N$ the function $w_{p}^{0}:=\frac{\partial}{\partial x_{p}} \widetilde{v}_{\xi}^{0}$ is the unique solution of the following problem

$$
\left\{\begin{array}{l}
\Delta_{x} w_{p}^{0}=0 \quad \text { if } x \in \Omega \\
w_{p}^{0}(x)=\frac{x_{p}-\xi_{p}}{|x-\xi|^{N}} \quad \text { if } x \in \partial \Omega .
\end{array}\right.
$$

We are interested in studying the non degeneracy of the critical points of the Robin function of the domain $\Omega_{\theta}$, namely by (5) and (8) the points $z \in \Omega_{\theta}$ such that

$$
0=\nabla_{z} t^{\Omega_{\theta}}(z)=\left.2 \nabla_{z} v_{y}^{\theta}(z)\right|_{y=z} .
$$

This is equivalent to study the non degeneracy of $x \in \Omega$ such that $0=\left.\nabla_{x} \widetilde{v}_{\xi}^{\theta}(x)\right|_{\xi=x}$. Thus, we are led to consider the map $F: \Omega \times \mathfrak{B}_{\rho} \rightarrow \mathbb{R}^{N}$ defined by

$$
F(x, \theta):=\left.\nabla_{x} \widetilde{v}_{\xi}^{\theta}(x)\right|_{\xi=x} .
$$

By Remark 2.3 and Lemma $4.2 F$ is a $C^{1}$-map.

We shall apply the following abstract transversality theorem to the map $F$ (see [13, 15, 16]). 
Theorem 2.4. Let $X, Y, Z$ be three Banach spaces and $U \subset X, V \subset Y$ open subsets. Let $F: U \times V \rightarrow Z$ be a $C^{\alpha}-$ map with $\alpha \geq 1$. Assume that

i) for any $y \in V, F(\cdot, y): U \rightarrow Z$ is a Fredholm map of index $l$ with $l \leq \alpha$;

ii) 0 is a regular value of $F$, i.e. the operator $F^{\prime}\left(x_{0}, y_{0}\right): X \times Y \rightarrow Z$ is onto at any point $\left(x_{0}, y_{0}\right)$ such that $F\left(x_{0}, y_{0}\right)=0$

iii) the map $\pi \circ i: F^{-1}(0) \rightarrow Y$ is $\sigma-$ proper, i.e. $F^{-1}(0)=\cup_{s=1}^{+\infty} C_{s}$ where $C_{s}$ is a closed set and the restriction $\pi \circ i_{\left.\right|_{s}}$ is proper for any $s$; here $i: F^{-1}(0) \rightarrow Y$ is the canonical embedding and $\pi: X \times Y \rightarrow Y$ is the projection.

Then the set $\Theta:=\{y \in V: 0$ is a regular value of $F(\cdot, y)\}$ is a residual subset of $V$, i.e. $V \backslash \Theta$ is a countable union of closet subsets without interior points.

Proof of the main result. We are going to apply the transversality theorem 2.4 to the map $F$ defined by (14). In this case we have $X=Z=\mathbb{R}^{N}, Y=\mathfrak{E}^{k}, U=\Omega \subset \mathbb{R}^{N}$ and $V=\mathfrak{B}_{\rho} \subset \mathfrak{E}^{k}$, where $\rho$ is small enough. Since $X=Z$ is a finite dimensional space, it is easy to check that for any $\theta \in \mathfrak{B}_{\rho}$, the map $x \rightarrow F(x, \theta)$ is a Fredholm map of index 0 and then assumption i) holds. As far as it concerns assumption iii), we have that

$$
F^{-1}(0)=\cup_{s=1}^{+\infty} C_{s}, \text { where } C_{s}:=\left\{\Omega_{s} \times \overline{\mathfrak{B}_{\rho-\frac{1}{s}}}\right\} \cap F^{-1}(0) \text { and } \Omega_{s}:=\{x \in \Omega: \operatorname{dist}(x, \partial \Omega) \leq 1 / s\} .
$$

Using the compactness of $\Omega_{s}$, we can show that the restriction $\pi \circ i_{\left.\right|_{C_{s}}}$ is proper, namely if the sequence $\left(\theta_{n}\right) \subset \overline{\mathfrak{B}_{\rho-\frac{1}{s}}}$ converges to $\psi_{0}$ and the sequence $\left(x_{n}\right) \subset \Omega_{s}$ is such that $F\left(x_{n}, \theta_{n}\right)=0$ then there exists a subsequence of $\left(x_{n}\right)$ which converges to $x_{0} \in \Omega_{s}$ and $F\left(x_{0}, \psi_{0}\right)=0$.

To prove that assumption ii) holds we will show in Lemma 3.1 that if $(\bar{x}, \bar{\theta}) \in \Omega \times \mathfrak{B}_{\rho}$ is such that $F(\bar{x}, \bar{\theta})=$ $\left.\nabla_{x} \widetilde{v}_{\xi}^{\bar{\theta}}(\bar{x})\right|_{\xi=\bar{x}}=0$ the map $F_{\theta}^{\prime}(\bar{x}, \bar{\theta}): \mathfrak{E}^{k} \rightarrow \mathbb{R}^{N}$ defined by $\left.\theta \rightarrow D_{\theta} \nabla_{x} \widetilde{v}_{\bar{x}}^{\theta}(x)\right|_{\theta=\bar{\theta}, x=\bar{x}}[\theta]$ is surjective.

Finally, we can apply the transversality theorem 2.4 and we get that the set

$$
\begin{aligned}
\mathfrak{A} & :=\left\{\theta \in \mathfrak{B}_{\rho}: F_{x}^{\prime}(x, \theta): \mathbb{R}^{N} \rightarrow \mathbb{R}^{N} \text { is invertible at any point }(x, \theta) \text { such that } F(x, \theta)=0\right\} \\
& =\left\{\theta \in \mathfrak{B}_{\rho}: \text { the critical points of the Robin function of the domain } \Omega_{\theta} \text { are nondegenerate }\right\}
\end{aligned}
$$

is a residual, and hence dense, subset of $\mathfrak{B}_{\rho}$.

\section{0 is a regular value of $F$}

In this section we show that 0 is a regular value of the map $F$ defined by (14).

Lemma 3.1. The map $\theta \rightarrow F_{\theta}^{\prime}(\bar{x}, \bar{\theta})[\theta]$ is onto on $\mathbb{R}^{N}$ for any $(\bar{x}, \bar{\theta}) \in \Omega \times \mathfrak{B}_{\rho}$ such that $F(\bar{x}, \bar{\theta})=0$.

Proof. Let us fix $(\bar{x}, \bar{\theta}) \in \Omega \times \mathfrak{B}_{\rho}$ such that $F(\bar{x}, \bar{\theta})=0$. We want to show that given $e^{(1)}, \ldots, e^{(N)}$ the canonical base in $\mathbb{R}^{N}$, for any $i=1, \ldots, N$ there exists $\theta \in \mathfrak{E}^{k}$ such that $F_{\theta}^{\prime}(\bar{x}, \bar{\theta})[\theta]=e^{(i)}$. We point out that the ontoness of the map $\theta \rightarrow F_{\theta}^{\prime}(\bar{x}, \bar{\theta})[\theta]$ is invariant with respect to the change of variables $\eta=(I+\bar{\theta})(x)$. We have that

$$
F_{\theta}^{\prime}(\bar{x}, \bar{\theta})[\theta]=\left(\frac{\partial}{\partial x_{1}} D_{\theta} \widetilde{v}_{\bar{x}}^{\bar{\theta}}[\theta](\bar{x}), \ldots, \frac{\partial}{\partial x_{N}} D_{\theta} \widetilde{v}_{\bar{x}}^{\bar{\theta}}[\theta](\bar{x})\right)
$$

because $\frac{\partial}{\partial x_{p}} D_{\theta} \widetilde{v_{\bar{x}}}[\theta](\bar{x})=D_{\theta} \frac{\partial}{\partial x_{p}} \widetilde{v}_{\bar{x}}^{\bar{\theta}}[\theta](\bar{x})$ as it is easy to verify.

Let $\bar{\eta}=\bar{x}+\bar{\theta}(\bar{x}) \in \Omega_{\bar{\theta}}$. By (8), (9), (10) and Lemma 4.2, we deduce that $v_{\bar{\eta}}^{\bar{\theta}}(\eta)=v_{\bar{\eta}}^{\bar{\theta}}(x+\bar{\theta}(x))=\widetilde{v}_{\bar{x}}^{\bar{\theta}}(x)$ is the unique solution of

$$
\left\{\begin{array}{l}
\Delta_{\eta} v_{\bar{\eta}}^{\bar{\theta}}=0 \quad \text { if } \eta \in \Omega_{\bar{\theta}} \\
v_{\bar{\eta}}^{\bar{\theta}}(\eta)=\Gamma(|\eta-\bar{\eta}|) \quad \text { if } \eta \in \partial \Omega_{\bar{\theta}} .
\end{array}\right.
$$


and $\left.\nabla_{\eta} v_{\bar{\eta}}^{\bar{\theta}}(\eta)\right|_{\eta=\bar{\eta}}=0$.

We consider the deformation $I+\bar{\theta}+\theta=(I+\alpha)(I+\bar{\theta})$, where $\theta=\alpha(I+\bar{\theta})$ and the domain $(I+\bar{\theta}+\theta) \Omega=$ $(I+\alpha)(I+\bar{\theta}) \Omega$. We set

$$
\bar{\eta}:=(I+\bar{\theta}) \bar{x} \quad \text { and } \quad \bar{z}:=(I+\alpha) \bar{\eta}
$$

Let $v_{\bar{z}}^{\bar{\theta}+\theta}$ be the unique solution of

$$
\left\{\begin{array}{l}
\Delta_{z} w(z)=0 \quad \text { if } z \in \Omega_{\bar{\theta}+\theta} \\
w(z)=\Gamma(|z-\bar{z}|) \quad \text { if } z \in \partial \Omega_{\bar{\theta}+\theta}
\end{array}\right.
$$

Then we set

$$
v_{\bar{z}}^{\bar{\theta}+\theta}(z)=v_{\bar{z}}^{\bar{\theta}+\theta}(\eta+\alpha(\eta))=\hat{v}_{\bar{\eta}}^{\bar{\theta}+\theta}(\eta)=\hat{v}_{\bar{\eta}}^{\bar{\theta}+\theta}(x+\bar{\theta}(x))=\widetilde{v}_{\bar{x}}^{\bar{\theta}+\theta}(x) .
$$

We immediately obtain that

$$
\left.D_{\theta} \hat{v}_{\bar{\eta}}^{\bar{\theta}+\theta}\right|_{\theta=0}[\beta](\eta)=\left.D_{\theta} \widetilde{v}_{\bar{x}}^{\bar{\theta}+\theta}\right|_{\theta=0}[\beta](x) \text { with } \eta=x+\bar{\theta}(x) .
$$

By (18) we have that given $\theta^{(1)}, \ldots, \theta^{(N)}$ the $N$ vectors

$$
\left.\nabla_{x} D_{\theta} \widetilde{v}_{\bar{x}}^{\bar{\theta}+\theta}\right|_{\theta=0}\left[\theta^{(1)}\right](\bar{x}), \ldots,\left.\nabla_{x} D_{\theta} \widetilde{v}_{\bar{x}}^{\bar{\theta}+\theta}\right|_{\theta=0}\left[\theta^{(N)}\right](\bar{x})
$$

are linearly independent if and only if the $N$ vectors

$$
\left.\nabla_{x} D_{\theta} \hat{v}_{\bar{\eta}}^{\bar{\theta}+\theta}\right|_{\theta=0}\left[\theta^{(1)}\right](\bar{\eta}), \ldots,\left.\nabla_{x} D_{\theta} \hat{v}_{\bar{\eta}}^{\bar{\theta}+\theta}\right|_{\theta=0}\left[\theta^{(N)}\right](\bar{\eta})
$$

are linearly independent.

At this stage our aim is to find $\theta^{(1)}, \ldots, \theta^{(N)}$ so that the $N$ vectors

$$
\left.\nabla_{x} D_{\theta} \hat{v}_{\bar{\eta}}^{\bar{\theta}+\theta}\right|_{\theta=0}\left[\theta^{(1)}\right](\bar{\eta}), \ldots,\left.\nabla_{x} D_{\theta} \hat{v}_{\bar{\eta}}^{\bar{\theta}+\theta}\right|_{\theta=0}\left[\theta^{(N)}\right](\bar{\eta})
$$

are linearly independent. First of all we point out that by Lemma 4.1 the function $w_{\bar{\eta}}^{\bar{\theta}}[\alpha](\cdot):=\left.D_{\theta} \hat{v}_{\bar{\eta}}^{\bar{\theta}+\theta}\right|_{\theta=0}[\theta](\cdot)$ is the unique solution of the problem

$$
\begin{cases}\Delta_{\eta} w-\sum_{i, j=1}^{N} \frac{\partial^{2} \hat{v}_{\bar{\eta}}^{\bar{\theta}}}{\partial \eta_{i} \partial \eta_{j}}(\eta)\left[\frac{\partial \alpha_{j}}{\partial \eta_{i}}(\eta)+\frac{\partial \alpha_{i}}{\partial \eta_{j}}(\eta)\right]-\sum_{j=1}^{N} \frac{\partial \hat{v}_{\bar{\eta}}^{\bar{\theta}}}{\partial \eta_{j}}(\eta) \Delta_{\eta} \alpha_{j}(\eta)=0 & \text { if } \eta \in \Omega_{\bar{\theta}} \\ w(\eta)=\sum_{i=1}^{N} \frac{\eta_{i}-\bar{\eta}_{i}}{|\eta-\bar{\eta}|^{N}}\left(\alpha_{i}(\eta)-\alpha_{i}(\bar{\eta})\right) & \text { if } \eta \in \partial \Omega_{\bar{\theta}}\end{cases}
$$

Here $\alpha=\theta(I+\bar{\theta})^{-1}$ and $\hat{v}_{\bar{\eta}}^{\bar{\theta}}$ is the unique solution of

$$
\left\{\begin{array}{lr}
\Delta_{\eta} \hat{v}_{\bar{\eta}}^{\bar{\theta}}=0 & \text { if } \eta \in \Omega_{\bar{\theta}} \\
\hat{v}_{\bar{\eta}}^{\bar{\theta}}(\eta)=\Gamma(|\eta-\bar{\eta}|) & \text { if } \eta \in \partial \Omega_{\bar{\theta}} .
\end{array}\right.
$$

We remark that by standard regularity theory (see also Remark 2.3) it follows

$$
\left\|\hat{v}_{\bar{\eta}}^{\bar{\theta}}\right\|_{C^{3}\left(\bar{\Omega}_{\bar{\theta}}\right)} \leq c(\bar{\theta}, \bar{\eta})
$$

for some positive constant depending only on $\bar{\theta}$ and $\bar{\eta}$.

Moreover, we also get that the function $\left.\eta \rightarrow \frac{\partial}{\partial \eta_{p}} D_{\theta} \hat{v}_{\bar{\eta}}^{\bar{\theta}+\theta}\right|_{\theta=0}[\theta](\eta)=\frac{\partial}{\partial \eta_{p}} w_{\bar{\eta}}^{\bar{\theta}}[\alpha](\eta)$ for $p=1, \ldots, N$ is the unique solution of the problem

$$
\left\{\begin{array}{l}
\Delta_{\eta} \frac{\partial}{\partial \eta_{p}} w_{\bar{\eta}}^{\bar{\theta}}[\alpha](\eta)-\frac{\partial}{\partial \eta_{p}}\left\{\sum_{i, j=1}^{N} \frac{\partial^{2} \hat{v}_{\bar{\eta}}^{\bar{\theta}}}{\partial \eta_{i} \partial \eta_{j}}(\eta)\left[\frac{\partial \alpha_{j}}{\partial \eta_{i}}(\eta)+\frac{\partial \alpha_{i}}{\partial \eta_{j}}(\eta)\right]\right\}-\frac{\partial}{\partial \eta_{p}}\left\{\sum_{j=1}^{N} \frac{\partial \hat{v}_{\bar{\eta}}^{\bar{\theta}}}{\partial \eta_{j}}(\eta) \Delta_{\eta} \alpha_{j}(\eta)\right\}=0 \text { if } \eta \in \Omega_{\bar{\theta}} \\
\frac{\partial}{\partial \eta_{p}} w_{\bar{\eta}}^{\bar{\theta}}[\alpha](\eta)=\frac{\partial}{\partial \eta_{p}}\left\{\sum_{i=1}^{N} \frac{\eta_{i}-\bar{\eta}_{i}}{|\eta-\bar{\eta}|^{N}}\left(\alpha_{i}(\eta)-\alpha_{i}(\bar{\eta})\right)\right\} \text { if } \eta \in \partial \Omega_{\bar{\theta}} .
\end{array}\right.
$$


Therefore we look for $\alpha^{(1)}, \ldots, \alpha^{(N)}$ such that the $N$ vectors

$$
\nabla_{\eta} w_{\bar{\eta}}^{\bar{\theta}}\left[\alpha^{(1)}\right](\bar{\eta}), \ldots, \nabla_{\eta} w_{\bar{\eta}}^{\bar{\theta}}\left[\alpha^{(N)}\right](\bar{\eta})
$$

are linearly independent. Using the Green's representation formula by (21) we get

$$
\begin{aligned}
& \frac{\partial}{\partial \eta_{p}} w_{\bar{\eta}}^{\bar{\theta}}[\alpha](\bar{\eta})=\int_{\partial \Omega_{\bar{\theta}}} \frac{\partial}{\partial \eta_{p}}\left\{\sum_{i=1}^{N} \frac{\eta_{i}-\bar{\eta}_{i}}{|\eta-\bar{\eta}|^{N}}\left(\alpha_{i}(\eta)-\alpha_{i}(\bar{\eta})\right)\right\} \frac{\partial G}{\partial \nu}(\eta, \bar{\eta}) d \sigma \\
& +\int_{\Omega_{\bar{\theta}}} \frac{\partial}{\partial \eta_{p}}\left\{\sum_{i, j=1}^{N} \frac{\partial^{2} \hat{v}_{\bar{\eta}}^{\bar{\theta}}}{\partial \eta_{i} \partial \eta_{j}}(\eta)\left[\frac{\partial \alpha_{j}}{\partial \eta_{i}}(\eta)+\frac{\partial \alpha_{i}}{\partial \eta_{j}}(\eta)\right]-\sum_{j=1}^{N} \frac{\partial \hat{v}_{\bar{\eta}}^{\bar{\theta}}}{\partial \eta_{j}}(\eta) \Delta_{\eta} \alpha_{j}(\eta)\right\} G(\eta, \bar{\eta}) d \eta .
\end{aligned}
$$

We now choose $\alpha^{(1)}$ so that

$$
\alpha_{1}^{(1)}(\eta)=|\eta-\bar{\eta}|^{N} \chi\left(\left[\operatorname{dist}\left(\eta, \partial \Omega_{\bar{\theta}}\right)\right]^{a}\right) \text { and } \alpha_{2}^{(1)}(\eta)=\cdots=\alpha_{N}^{(1)}(\eta)=0 .
$$

Since $\partial \Omega_{\bar{\theta}}$ is smooth, the function $\eta \rightarrow \operatorname{dist}\left(\eta, \partial \Omega_{\bar{\theta}}\right)$ is of class $C^{3}$ when $\eta$ is close enough to the boundary. Here the cut off function $\chi$ is of class $C^{3}$ and satisfies

$$
\chi(s)=1 \text { if } s \in(0, \bar{\rho}), \chi(s)=0 \text { if } s \in(2 \bar{\rho}, \infty),\left|\chi^{\prime}(s)\right| \leq \frac{1}{\bar{\rho}},\left|\chi^{\prime \prime}(s)\right| \leq \frac{1}{\bar{\rho}^{2}},\left|\chi^{\prime \prime \prime}(s)\right| \leq \frac{1}{\bar{\rho}^{3}}
$$

where $\bar{\rho}>0$ is such that $4 \bar{\rho} \leq \operatorname{dist}\left(\bar{\eta}, \partial \Omega_{\bar{\theta}}\right)$ and $\bar{\rho}$ will be chosen small enough. The positive number $a$ will be chosen $a \geq 4$ (so that estimate (30) holds).

By the definition of $\alpha^{(1)}$ and (22) we have

$$
\int_{\partial \Omega_{\bar{\theta}}} \frac{\partial}{\partial \eta_{p}}\left\{\sum_{i=1}^{N} \frac{\eta_{i}-\bar{\eta}_{i}}{|\eta-\bar{\eta}|^{N}}\left(\alpha_{i}(\eta)-\alpha_{i}(\bar{\eta})\right)\right\} \frac{\partial G}{\partial \nu}(\eta, \bar{\eta}) d \sigma=\int_{\partial \Omega_{\bar{\theta}}} \frac{\partial}{\partial \eta_{p}}\left(\eta_{1}-\bar{\eta}_{1}\right) \frac{\partial G}{\partial \nu}(\eta, \bar{\eta}) d \sigma=\delta_{1 p} \int_{\partial \Omega_{\bar{\theta}}} \frac{\partial G}{\partial \nu}(\eta, \bar{\eta}) d \sigma
$$

Moreover we have

$$
\begin{aligned}
& \int_{\Omega_{\bar{\theta}}} \frac{\partial}{\partial \eta_{p}}\left\{\sum_{i, j=1}^{N} \frac{\partial^{2} \hat{v}_{\bar{\eta}}^{\bar{\theta}}}{\partial \eta_{i} \partial \eta_{j}}(\eta)\left[\frac{\partial \alpha_{j}}{\partial \eta_{i}}(\eta)+\frac{\partial \alpha_{i}}{\partial \eta_{j}}(\eta)\right]-\sum_{j=1}^{N} \frac{\partial \hat{v}_{\bar{\eta}}^{\bar{\theta}}}{\partial \eta_{j}}(\eta) \Delta_{\eta} \alpha_{j}(\eta)\right\} G(\eta, \bar{\eta}) d \eta \\
& =\int_{\Omega_{\overline{\bar{\theta}}}^{\bar{\rho}}} \frac{\partial}{\partial \eta_{p}}\left\{2 \frac{\partial^{2} \hat{v}_{\bar{\eta}}^{\bar{\theta}}}{\partial \eta_{1}^{2}}(\eta) \frac{\partial \alpha_{1}^{(1)}}{\partial \eta_{1}}(\eta)-\frac{\partial \hat{v}_{\bar{\eta}}^{\bar{\theta}}}{\partial \eta_{1}}(\eta) \Delta_{\eta} \alpha_{1}^{(1)}(\eta)\right\} G(\eta, \bar{\eta}) d \eta=: \sigma_{p}^{(1)}(\bar{\rho}),
\end{aligned}
$$

where $\Omega_{\bar{\theta}}^{\bar{\rho}}:=\left\{\eta \in \Omega_{\bar{\theta}}: \operatorname{dist}\left(\eta, \partial \Omega_{\bar{\theta}}\right)<2 \bar{\rho}\right\}$.

We now establish an accurate estimate of $\sigma^{(1)}(\bar{\rho})$. By Lemma 3.2 proved at the end of this section, for $\bar{\rho}$ small enough we have that there exists $c_{1}>0$ such that

$$
|G(\eta, \bar{\eta})| \leq c_{1} \bar{\rho} \text { for any } \eta \in \Omega_{\bar{\theta}}^{\bar{\rho}} .
$$

Moreover, it is easy to check that there exists $c_{2}>0$ such that for any $t=\left(t_{1}, \ldots, t_{N}\right)$ with $|t| \leq 3$

$$
\left|\frac{\partial^{t}|\eta-\bar{\eta}|^{N}}{\partial \eta_{1}^{t_{1}} \cdots \partial \eta_{N}^{t_{N}}}\right| \leq\left\{c_{2} \text { if } N \geq 3, c_{2} \bar{\rho}^{-1} \text { if } N=2\right\} \quad \text { for any } \eta \in \Omega_{\bar{\theta}}^{\bar{\rho}} \text {. }
$$

By (25), (26), (27) and (20) it follows that

$$
\begin{aligned}
& \sigma_{p}^{(1)}(\bar{\rho}) \leq c \int_{\Omega_{\bar{\theta}}^{\bar{\rho}}}\left\{\left|\frac{\partial \alpha_{1}^{(1)}}{\partial \eta_{1}}\right|+\left|\frac{\partial^{2} \alpha_{1}^{(1)}}{\partial \eta_{1} \partial \eta_{p}}\right|+\left|\Delta_{\eta} \alpha_{1}^{(1)}\right|+\left|\frac{\partial}{\partial \eta_{p}} \Delta_{\eta} \alpha_{1}^{(1)}\right|\right\} d \eta \\
& \leq c \int_{\Omega_{\bar{\theta}}^{\bar{\rho}}}^{\left\{\left|\frac{\partial}{\partial \eta_{1}} \chi\left(d^{a}(\eta)\right)\right|+\left|\frac{\partial^{2}}{\partial \eta_{1} \partial \eta_{p}} \chi\left(d^{a}(\eta)\right)\right|+\left|\Delta_{\eta} \chi\left(d^{a}(\eta)\right)\right|+\left|\frac{\partial}{\partial \eta_{p}} \Delta_{\eta} \chi\left(d^{a}(\eta)\right)\right|\right\}} d \eta \\
& A_{p}(\eta)
\end{aligned}
$$


where $d^{a}(\eta):=\left[\operatorname{dist}\left(\eta, \partial \Omega_{\bar{\theta}}\right)\right]^{a}$.

Let us estimate $A_{p}(\eta)$ when $\eta \in \Omega_{\bar{\theta}}^{\bar{\rho}}$. We recall that $0 \leq d(\eta) \leq \bar{\rho}$ since $\eta \in \Omega_{\bar{\theta}}^{\bar{\rho}}$ and (23) holds. By a simple calculation of the derivatives of the function $\eta \rightarrow \chi\left(d^{a}(\eta)\right)$ we easily get that there exists $c_{3}>0$ such that

$$
0 \leq A_{p}(\eta) \leq c_{3}\left(\bar{\rho}^{a-2}+\bar{\rho}^{a-3}+\bar{\rho}^{a-4}+\bar{\rho}^{2 a-4}+\bar{\rho}^{2 a-5}+\bar{\rho}^{3 a-6}\right) \text { for any } \eta \in \Omega_{\bar{\theta}}^{\bar{\rho}} .
$$

Then choosing $a \geq 4$ we have that there exists $c_{4}>0$ such that

$$
0 \leq A_{p}(\eta) \leq c_{2} \text { for any } \eta \in \Omega_{\bar{\theta}}^{\bar{\rho}} .
$$

By (25), (26), (28) and (29) we deduce that $\lim _{\bar{\rho} \rightarrow 0} \sigma_{p}^{(1)}(\bar{\rho})=0$. Therefore, by (22), (24) and (25) we get

$$
\left.\nabla_{\eta} D_{\theta} \hat{v}_{\bar{\eta}}^{\bar{\theta}+\theta}\right|_{\theta=0}\left[\alpha^{(1)}\right](\bar{\eta})=\nabla_{\eta} w_{\bar{\eta}}^{\bar{\theta}}\left[\alpha^{(1)}\right](\bar{\eta})=\left(\sigma_{0}+\sigma_{1}^{(1)}(\bar{\rho}), \sigma_{2}^{(1)}(\bar{\rho}), \ldots, \sigma_{N}^{(1)}(\bar{\rho})\right),
$$

where $\sigma_{0}:=\int_{\partial \Omega_{\bar{\theta}}} \frac{\partial G}{\partial \nu}(\eta, \bar{\eta}) d \sigma \neq 0$.

In a similar way, for any $q=1, \ldots, N$ we can choose $\alpha^{(q)}$ such that

$$
\alpha_{q}^{(q)}(\eta)=|\eta-\bar{\eta}|^{N} \chi\left(\left[\operatorname{dist}\left(\eta, \partial \Omega_{\bar{\theta}}\right)\right]^{a}\right) \quad \text { and } \quad \alpha_{i}^{(q)}(\eta)=0 \text { if } i \neq q .
$$

Arguing as above, for any $q=1, \ldots, N$ we get

$$
\left.\nabla_{\eta} D_{\theta} \hat{v}_{\bar{\eta}}^{\bar{\theta}+\theta}\right|_{\theta=0}\left[\alpha^{(q)}\right](\bar{\eta})=\nabla_{\eta} w_{\bar{\eta}}^{\bar{\theta}}\left[\alpha^{(q)}\right](\bar{\eta})=(\sigma_{1}^{(q)}(\bar{\rho}), \ldots, \underbrace{\sigma_{0}+\sigma_{q}^{(q)}(\bar{\rho})}_{q-\text { th }}, \ldots, \sigma_{N}^{(q)}(\bar{\rho})),
$$

where $\lim _{\bar{\rho} \rightarrow 0} \sigma_{p}^{(q)}(\bar{\rho})=0$ for any $p=1, \ldots, N$.

Finally, we choose $\bar{\rho}$ small enough so that the $N$ vectors $\nabla_{\eta} w_{\bar{\eta}}^{\bar{\theta}}\left[\alpha^{(1)}\right](\bar{\eta}), \ldots, \nabla_{\eta} w_{\bar{\eta}}^{\bar{\theta}}\left[\alpha^{(N)}\right](\bar{\eta})$ are linearly independent and the claim follows.

Next, we prove Lemma 3.2 used in the proof of Lemma 3.1

Lemma 3.2. Given $y \in \Omega$, there exist $\tau_{0}>0$ and $c_{1}>0$ such that for any $\tau \in\left(0, \tau_{0}\right)$

$$
|G(x, y)| \leq c_{1} \tau \quad \forall x \in \Omega_{\tau}:=\{x \in \Omega \quad \operatorname{dist}(x, \partial \Omega) \leq \tau\} .
$$

Proof. Let us fix $y \in \Omega$. First of all, if $\tau$ is small enough, for any $x \in \Omega_{\tau}$ there exists a unique $p_{x} \in \partial \Omega$ such that

$$
\operatorname{dist}(x, \partial \Omega)=\left|x-p_{x}\right| \leq \tau .
$$

By mean value theorem we get for some $t \in(0,1)$

$$
G(x, y)=G(x, y)-G\left(p_{x}, y\right)=\left\langle\nabla_{x} G\left(t x+(1-t) p_{x}, y\right), x-p_{x}\right\rangle .
$$

Therefore, taking into account that (31) holds and also that $t x+(1-t) p_{x} \in \Omega_{\tau}$ for any $x \in \Omega_{\tau}$, we get

$$
|G(x, y)| \leq \tau \max _{x \in \Omega_{\tau}}\left|\nabla_{x} G(x, y)\right| .
$$

The claim will follow if we prove that

$$
\max _{x \in \Omega_{\tau}}\left|\nabla_{x} G(x, y)\right| \leq c(y)
$$

for some positive constant $c$ depending on $y$.

Let us recall that (see (10) $G(x, y)=\gamma[\Gamma(|x-y|)-H(x, y)]$. If we choose $\tau<\frac{\operatorname{dist}(y, \partial \Omega)}{2}$ then

$$
|x-y| \geq \operatorname{dist}(y, \partial \Omega)-\operatorname{dist}(x, \partial \Omega) \geq \frac{\operatorname{dist}(y, \partial \Omega)}{2}
$$


and so by the expression of $\Gamma$ in (2) we get

$$
\max _{x \in \Omega_{\tau}}\left|\nabla_{x} \Gamma(x, y)\right| \leq c(y)
$$

for some positive constant $c$ depending on $y$. Moreover, by (3) and by standard regularity theory (see Remark 2.3), we also have that

$$
\max _{x \in \Omega_{\tau}}\left|\nabla_{x} H(x, y)\right| \leq c(y)
$$

for some positive constant $c$ depending on $y$. Finally, by (33) and (34) and (11), we get (32) and so the claim is proved.

\section{The dependence on $\theta$ of $\widetilde{v}_{\xi}^{\theta}$ and $\nabla_{x} \widetilde{v}_{\xi}^{\theta}$}

In the following we calculate the Frechet derivative with respect to $\theta$ of $\widetilde{v}_{\xi}^{\theta}$ and $\frac{\partial}{\partial x_{p}} \widetilde{v}_{\xi}^{\theta}$ for $p=1, \ldots, N$. Moreover, we prove that the map $\theta \rightarrow \frac{\partial}{\partial x_{p}} \widetilde{v}_{\xi}^{\theta}$ is of class $C^{1}$ for any $p=1, \ldots, N$.

Lemma 4.1. For any $\xi \in \Omega$ the map $T: \mathfrak{B}_{\rho} \rightarrow C^{2, \alpha}(\bar{\Omega})$ defined by $T(\theta)=\widetilde{v}_{\xi}^{\theta}$ is of class $C^{1}$. Moreover

$$
T_{\theta}^{\prime}(0)[\theta]=\left.D_{\theta} \widetilde{v}_{\xi}^{\theta}\right|_{\theta=0}[\theta]=u[\theta]
$$

is the unique solution of the problem

$$
\begin{cases}\Delta_{x} u[\theta]+\sum_{i, j=1}^{N} \frac{\partial^{2} \widetilde{v}_{\xi}^{0}}{\partial x_{i} \partial x_{j}}\left[\frac{\partial \theta_{j}}{\partial x_{i}}+\frac{\partial \theta_{i}}{\partial x_{j}}\right]-\sum_{j=1}^{N} \frac{\partial \widetilde{v}_{\xi}^{0}}{\partial x_{j}} \Delta_{x} \theta_{j}=0 & \text { if } x \in \Omega \\ u[\theta](x)=-\sum_{i=1}^{N} \frac{x_{i}-\xi_{i}}{|x-\xi|^{N}}\left(\theta_{i}(x)-\theta_{i}(\xi)\right) & \text { if } x \in \partial \Omega\end{cases}
$$

Proof. First, we prove that the Gateaux derivative of the map $T$ at 0 is the unique solution of the problem (35).

It holds

$$
\left\{\begin{array}{cc}
\Delta_{x}\left(\frac{\widetilde{v}_{\xi}^{t \theta}-\widetilde{v}_{\xi}^{0}}{t}\right)+\sum_{i, j=1}^{N} \frac{\partial^{2}}{\partial x_{i} \partial x_{j}}\left(\widetilde{v}_{\xi}^{\theta}-\widetilde{v}_{\xi}^{0}\right) \frac{1}{t}\left[\frac{\partial \gamma_{j}^{t}}{\partial z_{i}}+\frac{\partial \gamma_{i}^{t}}{\partial z_{j}}+\sum_{s=1}^{N} \frac{\partial \gamma_{j}^{t}}{\partial z_{s}} \frac{\partial \gamma_{i}^{t}}{\partial z_{s}}\right] & \\
+\sum_{j=1}^{N} \frac{\partial}{\partial x_{j}}\left(\widetilde{v}_{\xi}^{\theta}-\widetilde{v}_{\xi}^{0}\right) \frac{1}{t} \sum_{s=1}^{N} \frac{\partial^{2} \gamma_{j}^{t}}{\partial z_{s}^{2}}+f^{t}=0 & \text { if } x \in \Omega \\
\left(\frac{\widetilde{v}_{\xi}^{t \theta}-\widetilde{v}_{\xi}^{0}}{t}\right)(x)=\frac{\Gamma(|x-\xi+t \theta(x)-t \theta(\xi)|)-\Gamma(|x-\xi|)}{t} & \text { if } x \in \partial \Omega,
\end{array}\right.
$$

where $\gamma^{t}$ is such that $I+\gamma^{t}=(I+t \theta)^{-1}$ so $\gamma^{t}(z)=-t \theta\left(z+\gamma^{t}(z)\right)$ and

$$
f^{t}:=\frac{1}{t}\left\{\sum_{i, j=1}^{N} \frac{\partial^{2} \widetilde{v}_{\xi}^{0}}{\partial x_{i} \partial x_{j}}\left[\frac{\partial \gamma_{j}^{t}}{\partial z_{i}}+\frac{\partial \gamma_{i}^{t}}{\partial z_{j}}+\sum_{s=1}^{N} \frac{\partial \gamma_{j}^{t}}{\partial z_{s}} \frac{\partial \gamma_{i}^{t}}{\partial z_{s}}\right]+\sum_{j=1}^{N} \frac{\partial \widetilde{v}_{\xi}^{0}}{\partial x_{j}} \sum_{s=1}^{N} \frac{\partial^{2} \gamma_{j}^{t}}{\partial z_{s}^{2}}\right\}
$$

By the fact that $(I+t \theta) \circ\left(I+\gamma^{t}\right)=I$ and by Remark 2.2 we deduce that

$$
\left(\gamma^{t}\right)^{\prime}(z)[h]=-t\left(I+t \theta^{\prime}(x)\right)^{-1}\left(\theta^{\prime}(x)[h]\right)
$$

and

$$
\left(\gamma^{t}\right)^{\prime \prime}(z)[h][k]=-t\left(I+t \theta^{\prime}(x)\right)^{-1}\left(\theta^{\prime \prime}(x)\left[h+\left(\gamma^{t}\right)^{\prime}(z)[h]\right]\right)\left[k+\left(\gamma^{t}\right)^{\prime}(z)[k]\right]
$$


where $x=z+\gamma^{t}(z)$. Then we get that as $t \rightarrow 0$

$$
f^{t} \rightarrow-\sum_{i, j=1}^{N} \frac{\partial^{2} \widetilde{v}_{\xi}^{0}}{\partial x_{i} \partial x_{j}}\left[\frac{\partial \theta_{j}}{\partial x_{i}}+\frac{\partial \theta_{i}}{\partial x_{j}}\right]-\sum_{j=1}^{N} \frac{\partial \widetilde{v}_{\xi}^{0}}{\partial x_{j}} \sum_{s=1}^{N} \frac{\partial^{2} \theta_{j}}{\partial x_{s}^{2}} \text { in } C^{0, \alpha}(\bar{\Omega}),
$$

because $\left\|\widetilde{v}_{\xi}^{\theta}-\widetilde{v}_{\xi}^{0}\right\|_{C^{2, \alpha}(\bar{\Omega})} \rightarrow 0$. Recalling that for $x \neq \xi$ we have

$$
\lim _{t \rightarrow 0} \frac{\Gamma(|x-\xi+t \theta(x)-t \theta(\xi)|)-\Gamma(|x-\xi|)}{t}=-\sum_{i=1}^{N} \frac{x_{i}-\xi_{i}}{|x-\xi|^{N}}\left(\theta_{i}(x)-\theta_{i}(\xi)\right),
$$

by (36) and (37), using the standard regularity theory, we get that $\left\|\frac{\widetilde{v}_{\xi}^{t \theta}-\widetilde{v}_{\xi}^{0}}{t}\right\|_{C^{2, \alpha}(\bar{\Omega})}$ is bounded. Then for any sequence $\left(t_{n}\right)$ such that $t_{n} \rightarrow 0$, the sequence of functions $\frac{\widetilde{v}_{\xi}^{t_{\xi} \theta}-\widetilde{v}_{\xi}^{0}}{t_{n}}$, up to a subsequence, is convergent in $C^{2}(\bar{\Omega})$ and by (36) and (37) it converges to the unique solution $u[\theta]$ of problem (35). In fact, by Remark 2.1 we have that

$$
\left\|\sum_{i, j=1}^{N} \frac{\partial^{2} \frac{\widetilde{v}_{\xi}^{\theta}-\widetilde{v}_{\xi}^{0}}{t}}{\partial x_{i} \partial x_{j}}\left[\frac{\partial \gamma_{j}^{t}}{\partial z_{i}}+\frac{\partial \gamma_{i}^{t}}{\partial z_{j}}+\sum_{s=1}^{N} \frac{\partial \gamma_{j}^{t}}{\partial z_{s}} \frac{\partial \gamma_{i}^{t}}{\partial z_{s}}\right]+\sum_{j=1}^{N} \frac{\partial \frac{\widetilde{v}_{\xi}^{\theta}-\widetilde{v}_{\xi}^{0}}{t}}{\partial x_{j}} \sum_{s=1}^{N} \frac{\partial^{2} \gamma_{j}^{t}}{\partial z_{s}^{2}}\right\|_{C^{0, \alpha}(\bar{\Omega})} \rightarrow 0
$$

as $t \rightarrow 0$. Next, it is easy to check that the Gateaux derivative exists and is continuous. Then the claim follows. (35).

Lemma 4.2. Let $p=1, \ldots, N$. For any $\xi \in \Omega$ the map $G: \mathfrak{B}_{\rho} \rightarrow C^{2, \alpha}(\bar{\Omega})$ defined by $G(\theta)=\frac{\partial \widetilde{v}_{\xi}^{\theta}}{\partial x_{p}}$ is of class $C^{1}$. Moreover

$$
G_{\theta}^{\prime}(0)[\theta]=\left.D_{\theta} \frac{\partial \widetilde{v}_{\xi}^{\theta}}{\partial x_{p}}\right|_{\theta=0}[\theta]=u_{p}[\theta]
$$

is the unique solution of the problem

$$
\begin{cases}\Delta_{x} u_{p}[\theta]-\frac{\partial}{\partial x_{p}} \sum_{i, j=1}^{N} \frac{\partial^{2} \widetilde{v}_{\xi}^{0}}{\partial x_{i} \partial x_{j}}\left[\frac{\partial \theta_{j}}{\partial x_{i}}+\frac{\partial \theta_{i}}{\partial x_{j}}\right]-\frac{\partial}{\partial x_{p}} \sum_{j=1}^{N} \frac{\partial \widetilde{v}_{\xi}^{0}}{\partial x_{j}} \Delta_{x} \theta_{j}=0 & \text { if } x \in \Omega \\ u_{p}[\theta](x)=-\frac{\partial}{\partial x_{p}} \sum_{i=1}^{N} \frac{x_{i}-\xi_{i}}{|x-\xi|^{N}}\left(\theta_{i}(x)-\theta_{i}(\xi)\right) & \text { if } x \in \partial \Omega .\end{cases}
$$

Proof. First, we prove that the Gateaux derivative of the map $G$ at 0 is the unique solution of the problem (35). The function $\frac{w_{p}^{t \theta}-w_{p}^{0}}{t}$ is a solution of the problem

$$
\left\{\begin{array}{l}
\Delta_{x}\left(\frac{w_{p}^{t \theta}-w_{p}^{0}}{t}\right)+\sum_{i, j=1}^{N} \frac{\partial^{2}\left(w_{p}^{t \theta}-w_{p}^{0}\right)}{\partial x_{i} \partial x_{j}} \frac{1}{t}\left[\frac{\partial \gamma_{j}^{t}}{\partial z_{i}}+\frac{\partial \gamma_{i}^{t}}{\partial z_{j}}+\sum_{s=1}^{N} \frac{\partial \gamma_{i}^{t}}{\partial z_{s}} \frac{\partial \gamma_{j}^{t}}{\partial z_{s}}\right] \\
+\sum_{j=1}^{N} \frac{\partial\left(w_{p}^{t \theta}-w_{p}^{0}\right)}{\partial x_{j}} \frac{1}{t} \sum_{s=1}^{N} \frac{\partial^{2} \gamma_{i}^{t}}{\partial z_{s}^{2}}+\frac{1}{t} \mathfrak{f}^{t \theta}(x)+\frac{1}{t} \mathfrak{g}^{t \theta}(x)=0 \quad \text { if } x \in \Omega \\
\left(\frac{w_{p}^{t \theta}-w_{p}^{0}}{t}\right)(x)=\frac{\varphi_{p}^{t \theta}(x)}{t} \quad \text { if } x \in \partial \Omega,
\end{array}\right.
$$


where

$$
\begin{aligned}
\mathfrak{f}^{\theta}(x) & :=\sum_{i, j=1}^{N} \frac{\partial^{2} \widetilde{v}_{\xi}^{\theta}}{\partial x_{i} \partial x_{j}} \frac{\partial}{\partial x_{p}}\left[\frac{\partial \gamma_{j}}{\partial z_{i}}+\frac{\partial \gamma_{i}}{\partial z_{j}}+\sum_{s=1}^{N} \frac{\partial \gamma_{i}}{\partial z_{s}} \frac{\partial \gamma_{j}}{\partial z_{s}}\right]+\sum_{j=1}^{N} \frac{\partial \widetilde{v}_{\xi}^{\theta}}{\partial x_{j}} \frac{\partial}{\partial x_{p}} \sum_{s=1}^{N} \frac{\partial^{2} \gamma_{i}}{\partial z_{s}^{2}} \\
\mathfrak{g}^{\theta}(x) & :=\sum_{i, j=1}^{N} \frac{\partial^{2} w_{p}^{0}}{\partial x_{i} \partial x_{j}}\left[\frac{\partial \gamma_{j}}{\partial z_{i}}+\frac{\partial \gamma_{i}}{\partial z_{j}}+\sum_{s=1}^{N} \frac{\partial \gamma_{i}}{\partial z_{s}} \frac{\partial \gamma_{j}}{\partial z_{s}}\right]+\sum_{j=1}^{N} \frac{\partial w_{p}^{0}}{\partial x_{j}} \sum_{s=1}^{N} \frac{\partial^{2} \gamma_{i}}{\partial z_{s}^{2}} \\
\varphi_{p}^{\theta}(x) & :=\frac{x_{p}-\xi_{p}+\theta_{p}(x)-\theta_{p}(\xi)}{|x-\xi+\theta(x)-\theta(\xi)|^{N}}-\frac{x_{p}-\xi_{p}}{|x-\xi|^{N}}+\sum_{i=1}^{N} \frac{x_{i}-\xi_{i}+\theta_{i}(x)-\theta_{i}(\xi)}{|x-\xi+\theta(x)-\theta(\xi)|^{N}} \frac{\partial \theta_{i}}{\partial x_{p}}
\end{aligned}
$$

Moreover $\gamma^{t}$ is such that $I+\gamma^{t}=(I+t \theta)^{-1}$ so $\gamma^{t}(z)=-t \theta\left(z+\gamma^{t}(z)\right)$. We point out that $\mathfrak{f}^{t \theta}$ in (40) and $\mathfrak{g}^{t \theta}$ in (41) also contain $\gamma^{t}$. By Remark 2.2 we deduce that if $z:=x+\theta(x)$

$$
\gamma^{t}(z)=-t \theta(x)+\sum_{i \geq 2}(-1)^{i}(t \theta(x))^{i} \text { and }\left(\gamma^{t}\right)^{\prime}(z)=-t \theta^{\prime}(x)+\sum_{i \geq 2}(-1)^{i}\left(t \theta^{\prime}(x)\right)^{i} .
$$

Then we have

$$
\lim _{t \rightarrow 0}\left\|\frac{1}{t} \mathfrak{f}^{t \theta}+\frac{1}{t} \mathfrak{g}^{t \theta}+\sum_{i, j=1}^{N} \frac{\partial^{2} \widetilde{v}_{\xi}^{0}}{\partial x_{i} \partial x_{j}} \frac{\partial}{\partial x_{p}}\left[\frac{\partial \theta_{j}}{\partial x_{i}}+\frac{\partial \theta_{i}}{\partial x_{j}}\right]+\sum_{j=1}^{N} \frac{\partial \widetilde{v}_{\xi}^{0}}{\partial x_{j}} \frac{\partial}{\partial x_{p}} \Delta_{x} \theta_{j}\right\|_{C^{0, \alpha}(\bar{\Omega})}=0 .
$$

Since $\frac{w_{p}^{t \theta}-w_{p}^{0}}{t}$ solves problem (39), by estimate (44) we deduce that $\left\|\frac{w_{p}^{t \theta}-w_{p}^{0}}{t}\right\|_{C^{2, \alpha}(\bar{\Omega})}$ is bounded as $t \rightarrow 0$. Then for any sequence $\left(t_{n}\right)$ such that $t_{n} \rightarrow 0$, the sequence of functions $\frac{w_{p}^{t_{n} \theta}-w_{p}^{0}}{t_{n}}$, up to a subsequence, is convergent in $C^{2}(\bar{\Omega})$. Moreover, arguing as in Remark 2.3 we can prove that

$$
\left\|\frac{\partial \widetilde{v}_{\xi}^{\theta}}{\partial x_{p}}-\frac{\partial \widetilde{v}_{\xi}^{0}}{\partial x_{p}}\right\|_{C^{2, \alpha}(\bar{\Omega})} \rightarrow 0 \text { as }\|\theta\|_{k} \rightarrow 0
$$

Finally, by (45), (43) and (44), passing to the limit in (39) as $t \rightarrow 0$ we get the claim. Next, it is easy to check that the Gateaux derivative exists and is continuous. Then the claim follows.

\section{References}

[1] BAhri, A.; Li, Y.; ReY, O. On a variational problem with lack of compactness: the topological effect of the critical points at infinity. Calc. Var. Partial Differential Equations 3 (1995), no. 1, 67-93,

[2] Bandle, C.; Flucher, M. Harmonic radius and concentration of energy; hyperbolic radius and Liouville's equations $-\Delta U=e^{U}$ and $-\Delta U=U^{(n+2) /(n-2)}$. SIAM Rev. 38 (1996), no. 2, 191-238,

[3] Baraket, S.; Pacard, F. Construction of singular limits for a semilinear elliptic equation in dimension 2. Calc. Var. Partial Differential Equations 6 (1998), no. 1, 1-38.

[4] Caffarelli, L. A.; Friedman, A. Convexity of solutions of semilinear elliptic equations. Duke Math. J. 52 (1985), no. 2, 431-456.

[5] del Pino, M.; Kowalczyk, M.; Musso, M. Singular limits in Liouville-type equations. Calc. Var. Partial Differential Equations 24 (2005), no. 1, 47-81.

[6] Esposito, P.; Grossi, M.; Pistoia, A. On the existence of blowing-up solutions for a mean field equation. Ann. Inst. H. Poincaré Anal. Non Liné aire 22 (2005), no. 2, 227-257. 
[7] Ge, Y.; Jing, R.; Pacard, F. Bubble towers for supercritical semilinear elliptic equations. J. Funct. Anal. 221 (2005), no. 2, 251-302.

[8] Gilbarg, D.; Trudinger, N. S. Elliptic partial differential equations of second order. Grundlehren der Mathematischen Wissenschaften, Vol. 224. Springer-Verlag, Berlin-New York, 1977. x+401 pp.

[9] Gladiali, F.; Grossi, M. Some results for the Gelfand's problem. Comm. Partial Differential Equations 29 (2004), no. 9-10, 1335-1364.

[10] Grossi, M. On the nondegeneracy of the critical points of the Robin function in symmetric domains. $C$. R. Math. Acad. Sci. Paris 335 (2002), no. 2, 157-160

[11] Ma, L.; WeI, J. Convergence for a Liouville equation. Comment. Math. Helv. 76 (2001), no. 3, 506-514.

[12] Pistoia, A. On the uniqueness of solutions for a semilinear elliptic problem in convex domains.Differential Integral Equations 17 (2004), no. 11-12, 1201-1212.

[13] Quinn, F. Transversal approximation on Banach manifolds. 1970 Global Analysis (Proc. Sympos. Pure Math., Vol.XV, Berkeley, Calif., 1968) pp. 213-222 Amer. Math. Soc., Providence, R.I.

[14] REY, O. The role of the Green's function in a nonlinear elliptic equation involving the critical Sobolev exponent. J. Funct. Anal. 89 (1990), no. 1, 1-52.

[15] Saut, J.-C.; Temam, R. Generic properties of nonlinear boundary value problems. Comm. Partial Differential Equations 4 (1979), no. 3, 293-319.

[16] Uhlenbeck, K. Generic properties of eigenfunctions. Amer. J. Math. 98 (1976), no. 4, 1059-1078. 